UDK $502.7(24)$

Alf $G R U B E^{*}$

\title{
GEOTOPSCHUTZ ALS WICHTIGE AUFGABE DER GEOWISSENSCHAFTEN
}

\author{
Einleitung
}

Der Umwelt- und Naturschutz ist eines der vordringlichsten Ziele unserer Zeit. Der Schutz unserer Umwelt ist gleichzeitig Erhaltung der Lebensgrundlage des Menschen. Leider wurde der geowissenschaftliche Naturschutz bisher vernachlässigt. Die Geologie und Geomorphologie können nur in weit geringerem Maße als die Biologie von der „Liebe zur Natur" eines Großteils der Bevölkerung profitieren. Aber auch der Erhalt geowissenschaftlich schützenswerter Bereiche ist von wesentlicher Bedeutung für das öffentliche Interesse, die Ökonomie und die Wissenschaft.

Die Geschichte des Geotopschutzes reicht weit zurück. So gab es erste Ergebnisse des Geotopschutzes in Deutschland bereits im Jahre 1836. Damals wurde der Drachenfels, als eindrucksvolles Beispiel von tertiärem Vulkanismus (oligozäner Trachyt) am Rhein, das erste deutsche Naturschutzgebiet. 1852 wurde die Teufelsmauer, ein Härtlingszug nördlich des Harzes unter Schutz gestellt.

\section{Aufgaben des Geotopschutzes}

Geotopschutz ist von Bedeutung für die Allgemeinheit, Ökonomie und Wissenschaft. Viele Arbeitsplätze hängen z. B. in Deutschland am Fossilienhandel, so in den Bereichen Solnhofen und Holzmaden, wo Fossilien als Einzelstücke oder auch zu mehreren Exemplaren in Platten gehandelt werden. Geotope bilden als Untersuchungsobjekt und „Datenträger" die Grundlage der wissenschaftlichen Forschung. Die genannte Lokalität Holzmaden bietet z. B. einmalige Exemplare des Ichtyosaurus, teilweise mit Embryonen, eingebettet in Unterjurassische Posidonienschiefer. Solnhofen mit seinen lithographischen Kalksteinen des Malms ist berühmt geworden für die Funde von Archäopterix. Es gibt auch eine Reihe von Beispielen, bei denen Geotope für die spätere Forschung von Bedeutung waren. Geotope sind wichtige Zeugen der Entstehung unseres Landes, sind landschaftsgeschichtliche „Nachschlagewerke", die der Nachwelt erhalten bleiben müssen. An ihnen können formende Prozesse rekonstruiert werden, die heute nicht mehr ablaufen. Sie sind zudem häufig auch von großem ästhetischem Wert. GeotopSchutz ist zudem für die Erziehung sehr wichtig; so ist die Kartierarbeit im Aufschluß ein wesentlicher Bestandteil geowissenschaftlicher Ausbildung.

* Geologisch-Paläontologisches Institut der Universität Kiel (Kieli Ulikooli GeoloogiaPaleontoloogia Instituut), Olshausenstrasse 40-60, 2300 Kiel, Deutschland. 
Ziel des Geotopschutzes ist die dauerhafte Sicherung einer repräsentativen Auswahl von geowissenschaftlichen Objekten und Formen sowie besonderen Einzelschöpfungen. Es gilt die wichtigsten Bereiche als Spiegelbild der Erd- und Menschheitsgeschichte zu erhalten. Für die Unterschutzstellung gibt es eine Reihe von Kategorien, die meist für biologische Objekte konzipiert wurden. In einigen Ländern, wie z. B. in Neuseeland, England und Frankreich gibt es Schutzgebiete speziell für geowissenschaftliche Objekte (Gonggrijp, Boekschoten, 1981; Grigorescu, Norman, 1990; Soyez, 1973).

\section{Begriffsklärung}

Geowissenschaftlicher Naturschutz umfasst die Gesamtheit der Maßnahmen zur Inventarisierung, Bewertung, zu dem Schutz und der Pflege von geowissenschaftlich wertvollen Bereichen. Dabei werden teilweise speziell aus dem Geowissenschaften stammende Methoden verwendet bzw. entwickelt. In weiten Bereichen existieren Verbindungen und Ubergänge zum biologischen Naturschutz.

Der Begriff Geotop wurde schon früh, allerdings in etwas abweichender Definition verwendet (Paffen, 1953). Im englischen Sprachraum ist der Begriff Geotop nicht gebräuchlich (Crowther, Wimbledon, 1988). In Deutschland ist bisher der Begriff GeoSchOb (Geowissenschaftlich schützenswerte Objekte) verwendet worden, der jedoch vermutlich weitgehend durch Geotop ersetzt werden wird (Ross, 1991).

Geotope sind definitionsgemäß Teile des unbelebten Erdkörpers, die von einheitlicher Beschaffenheit sind und sich so mehr oder weniger scharf von der Umgebung abgrenzen lassen. Sie besitzen häufig regionale oder lokale landschaftsgeschichtliche Relevanz. $\mathrm{Zu}$ den Geotopen gehören z. B. Landschaftseinheiten, Fossilfundstätten, geologische Profile, Kliffaufschlüsse usw. Auch gehören anthropogene Formen, wie z. B. ehemalige Bergwerke/Schaustollen und Subrosionssenken über Stollen, zu den schutzwürdigen Formen (German, 1974).

Ein System der Geotope würde z. B. stratigraphische, paläontologische, petrographische, tektonische, (paläo-) pedologische und hydrogeologische Geotope enthalten. Eine besondere Gruppe wären Impakt-Geotope, wie z. B. das Nördlinger Ries, welches im Miozän entstand und einen Durchmesser von ca. $25 \mathrm{~km}$ hat. Die Geomorphotope, d.h. schützenswerte geomorphologische Formen, könnten nach Formenkreisen (glazial, äolisch, fluvial, abrasiv, Karst usw.) gegliedert werden. Uberschneidungen zwischen Geotopen und Geomorphotopen würden in einigen Küstenbereichen Estlands existieren, wo wichtige paläontologische Fundstätten gleichzeitig eindrucksvolle Kliffs bilden. Durch Zusätze könnte angegeben werden, ob es sich um ein künstliches Geotop (z. B. Kiesgrube) oder ein natürliches Geotop (z. B. ein Kliff) handelt. Erkenntnishistorische Geotope sind Bereiche, in denen bedeutende Basisuntersuchungen oder Erkenntnisse gewonnen wurden. Ein Beispiel aus Deutschland ist der Scheibenberg mit seinen Basaltsäulen, wo A. G. Werner seine Ideen im Neptunisten-Plutonisten-Streit entwickelte.

\section{Europäische Initiativen zum Geotopschutz}

In den meisten europäischen Ländern existieren inzwischen Inventare zu geowissenschaftlich schützenswerten Objekten oder doch Ansätze hierzu. Während sich einige Länder, wie z. B. England, schon relativ früh und intensiv mit geowissenschaftlichem Naturschutz beschäftigten, 
waren in vielen Ländern ähnliche Hindernisse wie in Deutschland vorhanden. Daraus entstand der Gedanke, eine überregionale Arbeitsgruppe mit internationalen Kontakten aufzubauen. 1988 wurde der erste internationale Workshop zum Geotopschutz in Leersum (Niederlande) abgehalten; als Resultat wurde die „European Working Group on Earth Science Conservation" gegründet, welche auch eine eigene kleine Zeitung herausgibt (Andersen, 1990). Weitere Treffen wurden 1989 in Lochau nahe Bregenz (Österreich) und 1990 in Lom (Norwegen) abgehalten. 1991 schließlich fand das ,1st Internat. Symposium for the Protection of our Geological Heritage" in Digne (Provence) statt, an dem über 120 Geowissenschaftler aus mehr als 30 Staaten teilnahmen.

Von paläontologischer Seite ist vor kurzem die Einrichtung einer internationalen Arbeitsgruppe vorgeschlagen worden, die sich mit den Möglichkeiten einer europaweiten Regelung zur Kontrolle und zum Schutz von Fossilien beschäftigen will.

\section{Inventare und Rechtliche Aspekte}

Im folgenden soll auf die unterschiedlichen Techniken zur Inventarisierung und Bewertung von schutzwürdigen Objekten nicht genau eingegangen werden. Generell können systematische und nicht systematische Inventare unterschieden werden (für Deutschland siehe z. B. Becker-Platen, 1982; Händel, 1991; Wagenbreth, 1970). Eine grobe Kategorisierung umfasst vier Stufen: international, national, regional und lokal wertvolle Bereiche; die Bewertung erfolgt häufig nach einem Punktesystem.

Für international herausragende schutzwürdige Geotope steht die Verleihung des Standards eines "World Heritage Site" durch die UNESCO (United Nations Educational, Scientific and Cultural Organisation) zur Verfügung, wie sie auch für kulturelle und biologische Objekte existiert. Zur Erfüllung dieser Aufgabe wurde eine ,,Task ForceGruppe" gebildet, die Vorschläge für die genannte Liste erarbeitet. Diese Arbeiten sind noch nicht abgeschlossen (freundl. Mitt. W. A. Wimbledon; Peterborough, England) und die Liste ist noch unvollständig, da viele Länder noch keine Vorschläge gemacht haben. Auch von Estland werden Vorschläge für diese Liste erarbeitet werden. $\mathrm{Zu}$ diesen bedeutenden Objekten zählen sicher auch Profilabschnitte des Silurs und Ordoviziums aus Estland (Rõõmusoks, 1976).

Insgesamt sind viele tausend schutzwürdiger Objekte in Deutschland inventarisiert, von diesen ist jedoch nur ein kleiner Teil ausreichend geschützt. Der Verlust wertvoller Objekte durch Verfüllung, Gesteinsabbau, Bebauung usw. ist erheblich.

Insgesamt sind die gesetzlichen Möglichkeiten zum Schutz von geowissenschaftlich schützenswerten Bereichen in den meisten europäischen Ländern verbesserungsfähig. Aufgrund der sehr unterschiedlichen Gegebenheiten sollen die relevanten Gesetze in den verschiedenen europäischen Ländern nicht näher beschrieben werden.

In Deutschland wird von einigen Geowissenschaftlern ein eigenes Gesetz oder ein separater Teil im Bundesnaturschutzgesetz sowie in den Landesgesetzen gefordert. In einem derartigen Gesetz könnten auch Fragen des Zugangs von Geowissenschaftlern zu geschützten Objekten sowie eine gesetzlich festgeschriebene Inventarisierung von Geotopen in den Ländern geregelt werden. Zur Zeit können Wissenschaftler nach Unterschutzstellung eines Objektes nicht immer weitere wissenschaftliche Untersuchungen durchführen. 


\section{Zukunftsperspektive}

Trotz der engagierten Arbeit von vielen Geowissenschaftlern im Geotopschutz muß die Situation bei der Inventarisierung und Unterschutzstellung wichtiger geowissenschaftlich interessanter Bereiche in vielen europäischen Ländern als unzureichend bezeichnet werden.

Der Schutz von Geotopen darf nicht nur seltene und spektakuläre Objekte umfassen, sondern sollte ein repräsentatives Spektrum aller Formen enthalten.

Als dringliche Aufgaben stehen an erster Stelle: zügige Inventarisierung, Bewertung der Objekte unter Benutzung eines einheitlichen Systems; verläßlicher Schutz von Geotopen, Einbeziehung von Fossil- und Mineraliensammlungen in Schutzstrategien; Popularisierung des geowissenschaftlichen Naturschutzes in der Öfentlichkeit sowie Mitarbeit in europäischen und internationalen Gremien.

Es bleibt $\mathrm{zu}$ hoffen, daß in Zukunft auch dem geowissenschaftlichen Naturschutz größere Aufmerksamkeit geschenkt werden wird und Geotope verstärkt geschützt werden können. Jeder Geowissenschaftler kann hierzu etwas beitragen.

\section{I T E R A T U R}

Andersen, S. 1990. Earth-science conservation: An absolute need for science and education. - Jb. Geol. B.-A., 133, 653-669.

Becker-Platen, J. D. 1982. Zur Kartierung schutzwürdiger geowissenschaftlicher Objekte in Niedersachen. - Geowiss. Beiträge zum Naturschutz (Laufener Seminarbeiträge), 7, 44-57.

Crowther, R. P., Wimbledon, W. A. 1988. The use and conservation of palaeontological sites. - Special papers in Palaeontology, 40.

German, R. 1974. Das mittelfristige Programm zum Schutz geologisch besonders wichtiger Naturdenkmale in Baden-Württemberg. - Veröff. Landestelle Naturschutz und Landschaftspflege Baden-Württ., 42, 85-92.

Gonggrijp, G. P., Boekschoten, G. J. 1981. Earth-science conservation: No science without conservation. - Geol. en Mijnbouw, 60, 433-445.

Grigorescu, D., Norman, D. 1990. Earth-science conservation in Romania. - Earth Science Conservation NCC, Peterborough, 27, 6-8.

Händel, D. 1991. Konzeption für ein System Geologischer Naturdenkmale des Präkänozoikums. - Z. geol. Wiss., 19, 73-78.

Paffen, K. 1953. Die natürliche Landschaft und ihre räumliche Gliederung. - Forsch. z. dt. Landeskunde, 68.

Ross, $P$. 1991. Geologisch-geomorphologisch schützenswerte Objekte (GeoschOb) in Schleswig-Holstein 1:250 000. - Geol. Landesamt S.-H., Kiel.

Rõõmusoks, A. 1976. Eesti paleosoikumi stratotüüpsete paljandite kaitsest ja uuritusest. - Eesti NSV maapōue kaitsest. Tallinn, Valgus, 108-121.

Soyez, D. 1973. Geowissenschaften und Naturschutz in Schweden. - Rückblick und Entwicklungstendenzen. - Erdkunde, XXVII, 140-146.

Wagenbreth, O. 1970. Entwurf eines Systems geologischer Naturdenkmale in Thüringen. - Landespflege u. Natursch. Thüringen, $7(1 / 2), 5-19$.

Vorgelegt von A. Raukas

Eingegangen

am 2, März 1992 


\section{ELUTA LOODUSE KAITSE KUI GEOLOOGIATEADUSE OLULINE ULESANNE}

Keskkonna ja looduse kaitse on praegusaja olulisemaid ülesandeid. Eluta looduse ja loodusharulduste kaitse teaduslike probleemidega on aga seni vähe tegeldud. Oluliseks tuleb pidada nii geoloogiliste üksikobjektide (geoloogilised paljandid, fossiilide leiukohad jt.) kui ka piirkondade (karstivormid jt.) kaitse alla võtmist. Selliste objektide kohta tarvitatakse mõistet geotoop, mis tähendab ühesuguselt määratletavat eluta looduse osa, mida on võimalik ümbruskonnast välja eraldada. Geotoopide süsteem sisaldab stratigraafilisi, paleontoloogilisi, petrograafilisi, tektoonilisi, paleopedoloogilisi ja hüdrogeoloogilisi geotoope. Eraldi grupi moodustavad impaktgeotoobid. Geomorfotoop on kaitset vääriv geomorfoloogiline pinnavorm (glatsiaalne, eooliline või muu).

\section{Альф ГРУБЕ}

\section{ОХРАНА НЕЖИВОЙ ПРИРОДЫ - ВАЖНАЯ ЗАДАЧА ГЕОЛОГИЧЕСКИХ НАУК}

Охрана окружающей среды - это одна из важнейших проблем настоящего времени. Однако вопросы охраны уникальных объектов неживой природы до сих пор остаются без должного внимания. Вводится в оборот термин «геотоп», под которым понимается часть неживой природы с только ей присущими свойствами. В частности, геоморфотопами являются формы рельефа. 